\title{
Evaluación nutricional y sensorial de un alimento a base de atún y soya enriquecido con vitaminas y minerales
}

\author{
Nutritional and sensorial evaluation \\ of a food with tuna and soya \\ enriched with vitamins and minerals
}

\begin{abstract}
The objective of this research was to determine the nutritional and sensorial impact of a food with tuna and soya enriched with vitamins and minerals in pre-school children of three communities of Chiapas, Mexico. It is a study of nutritional intervention with a cohorte of six months. The nutritional state of children was determined previuos to the nurishing complement, to the half and at the end of this. The sample was conformed with the children who presented undernourishment problems; they were fed during six months. The level of acceptability of the food was determined with the children in pre-school age of the three communities. Significant evidences $(p<0.05)$ of the high level of acceptability of the food, when comparing the obtained nutritional state at the beginning, half and at the end of the study were detected. Improvement on the health state was observed, when their severe malnutrition normalized.
\end{abstract}

Key words: undernourishment; nourishing intervention, preschool children, micronutrients, fortified food.

\section{INTRODUCCIÓN}

La desnutrición es el estado patológico sistémico y potencialmente reversible que se genera por el aporte insuficiente de nutrientes, o por una alteración en su utilización por las células del organismo. La desnutrición junto con la pobreza y el subdesarrollo, constituyen un círculo vicioso del cual es difícil desprenderse; sumidas en estas adversas condiciones vive casi un tercio de la población mundial (1). El estado nutricional, es un indicador clave de pobreza y salud, y su reconocimiento es importante para mejorar las políticas públicas de salud y de desarrollo global (2). Los niños son los más afectados por este problema; la tercera parte de las muertes en niños ocurre por problemas de desnutrición y la mayor prevalencia ocurre en países con baja y mediana ingesta de alimentos (3). Entre los factores que conllevan a que los niños sufran desnutrición, están la baja ingesta dietaría, la inaccesibilidad e inadecuada distribución de los alimentos al interior del hogar, la inapropiada preparación y almacenamiento de los alimentos, y las enfermedades infecciosas.

La deficiencia de micronutrientes es considerado un problema de salud pública $(4,5)$, especialmente para los
Laura Concepción Cruz-Domínguez Brenda Angélica Utrilla-Estrada Leonides Elena Flores-Guillén Esmeralda García-Parra Erika Judith López-Zúñiga Gilber Vela-Gutiérrez

Laboratorio de Investigación y Desarrollo de Productos Funcionales. Facultad de Ciencias de la Nutrición y Alimentos. Universidad de Ciencias y Artes de Chiapas (UNICACH). México.

Dirigir la correspondencia a: Profesor

Gilber Vela-Gutierrez Facultad de Ciencias de la Nutrición y Alimentos. UNICACH. Tel. (52)961 6170440 Ext. 4267/4260. E-mail: gilber.vela@unicach.mx

Este trabajo fue recibido el 8 de Marzo de 2016 y aceptado para ser publicado el 5 de Noviembre de 2016.

infantes y niños, debido a la ingesta inadecuada o utilización ineficiente de los alimentos (6). En el mundo existe alrededor de dos billones de personas que tienen alguna deficiencia de micronutrientes por ejemplo, anemia causada por deficiencia de hierro $(6,7)$. También se presenta un incremento en la tasa de infección y mortalidad causado por la deficiencia de vitamina $A$ y zinc, que son amenazas para el desarrollo de los niños (6). Según datos de la ENSANUT (8), la deficiencia de hierro y vitamina en niños menores de 5 años en México sigue siendo un problema de salud pública, debido a su contribución en los procesos de regulación metabólica. De acuerdo a datos de la Encuesta Nacional de Salud y Nutrición (ENSANUT) (8), en México $2.8 \%$ de los niños menores de cinco años presentan bajo peso, $13.6 \%$ baja talla y $1.6 \%$ emaciación. Datos de la misma encuesta, reporta que el sur de México se sigue manteniendo, como ha sucedido históricamente, las mayores prevalencias de baja talla (19.2\%), siendo las localidades rurales del sur las más afectadas, con una prevalencia de 13.9 puntos porcentuales (pp) mayor que el promedio nacional (13.6\%). Para el estado de Chiapas, las prevalencias de bajo peso es $8.4 \%$, la de baja talla $31.4 \%$ y 
la emaciación $2.4 \%(8)$.

Una de las alternativas para resolver este problema son los alimentos enriquecidos, que se definen como la adición de una o varias vitaminas, minerales o proteínas (aminoácidos) en concentraciones superiores a los que normalmente contiene el producto. Existen alimentos ricos en proteínas tanto de origen animal como vegetal que coadyuvan a mejorar los perfiles de biodisponibilidad favoreciendo el mantenimiento y mejora del estado de nutrición de la población.

En México el Instituto de Desarrollo Integral de la Familia (DIF), opera programas de ayuda alimentaria en poblaciones vulnerables; específicamente en Chiapas ha utilizado un producto diseñado a base de atún y soya, sin embargo, se desconoce los efectos en la recuperación del estado de nutricional de los menores de 5 años que han sido beneficiados con este programa. El objetivo de la presente investigación fue determinar el impacto nutricional de dicho alimento, elaborado a base de atún y soya, enriquecido con vitaminas y minerales en preescolares de cuatro jardines de niños de tres comunidades del municipio de Jiquipilas; Chiapas, México.

\section{SUJETOS Y MÉTODOS}

El presente estudio es prospectivo, no experimental, de intervención con una cohorte de seis meses. Mediante pruebas antropométricas se determinó el estado nutricional de niños en edad preescolar (3 y 5 años y nueve meses) de cuatro jardines de niños, posteriormente se complementó la dieta durante seis meses a los que presentaron problemas de desnutrición (grupo en estudio), y se brindó seguimiento durante ese periodo al resto de los niños.

\section{Consideraciones bioéticas}

Previo a la puesta en marcha, el presente estudio fue revisado por el Comité de Investigación, y validado posteriormente por el Comité de Bioética (FWA00017088), ambos de la Facultad de Ciencias de la Nutrición y Alimentos de la Universidad de Ciencias y Artes de Chiapas. A cada uno de los padres de familia de los niños participantes se les explicó en detalle en qué consistía el proyecto, posteriormente firmaron el consentimiento informado; éste y otros documentos confidenciales se mantienen bajo resguardo.

\section{Participantes}

Se conformó por 121 niños en edad preescolar pertenecientes a cuatro jardines de niños de tres comunidades rurales (Julian Grajales, Tierra y Libertad y Michoacán) del municipio de Jiquipilas Chiapas en México.

\section{Muestreo y muestra}

El muestreo fue no probabilístico a conveniencia, debido a que se realizó un diagnóstico nutricional previo a la intervención (complementación alimentaria), donde se obtuvieron 51 niños con problemas de desnutrición, los que conformaron la muestra (grupo en estudio); paralelamente se monitoreó al resto de los infantes que no manifestaron problemas de mal nutrición (70 niños) y quiénes conformaron el grupo control.

Criterios de inclusión, exclusión y eliminación

Criterios de inclusión: Niños en edad preescolar, inscritos en cualquiera de los cuatro jardínes de niños participantes, que no presentaron intolerancia a productos marinos y a la soya, que contaron con la autorización del padre de familia o tutor (consentimiento informado firmado).
Criterios de exclusión: Niños que a pesar de la autorización del padre o tutor decidieron no participar, o previo al estudio presentaban alguna patología que impidió su participación.

Criterios de eliminación: Niños que se enfermaron durante el estudio, o cambiaron de residencia o de jardín de niños.

\section{Descripción del alimento}

El producto fue elaborado por una empresa comercial, se formuló a base de atún (aleta amarilla), soya y enriquecido con vitaminas (ácido fólico, vitaminas A, B6, B12, C, D y E) y minerales (hierro y zinc); una porción de $60 \mathrm{~g}$ del alimento contenía, $10 \mathrm{~g}$ de proteínas, $5 \mathrm{~g}$ de lípidos totales, 0,94 g de carbohidratos y $232 \mathrm{mg}$ de sodio, y aportan $370 \mathrm{kj}(89 \mathrm{Kcal})$.

\section{DESARROLLO DE LA INVESTIGACIÓN \\ Evaluación sensorial}

Previo a la intervención nutricional, se realizó una prueba de evaluación sensorial con los 121 niños (jueces no entrenados) pertenecientes a los cuatro jardínes de niños de las tres comunidades. La prueba consistió en otorgarle a cada niño una muestra del alimento y se les pidió que lo consumieran, posteriormente se les indicó que registraran en una boleta (estructurada con caritas de emoción) el nivel de agrado de acuerdo a su percepción: Agrado (carita feliz), neutral (carita indiferente) y desagrado (carita triste). Los datos se registraron y compararón de acuerdo al nivel de agrado en las tres comunidades.

\section{Evaluación nutricional}

Inicialmente se realizó un diagnóstico nutricional al total de los niños inscritos en los cuatro jardines de niños $(m=121)$ de las tres comunidades; en esta evaluación se determinó el peso utilizando una báscula de piso (Seca®, Modelo 762, Germany) con una capacidad máxima de $150 \pm 0,5 \mathrm{~kg}$; la talla con un estadímetro (Seca $\circledR_{1}$, Modelo 213, Germany) con un intervalo de medición de 20 a 205 $\pm 0,05 \mathrm{~cm}$; la edad y el sexo de los infantes (9), que sirvió para obtener los indicadores de peso para la talla $(\mathrm{P} / \mathrm{T})$, peso para la edad $(\mathrm{P} / \mathrm{E})$ y talla para la edad (T/E), para su correlación con las Tablas de la National Survey of Children's Health (NSCH) y así poder diagnosticar el estado nutricional de los niños. Luego, se eligieron dos grupos (grupo 1 y 2). El grupo 1, estuvo conformado por 51 niños, quienes manifestaron problemas de desnutrición y que cumplieron con los criterios de inclusión; y el grupo 2, fue constituido por 70 niños que no presentaron problemas de malnutrición.

Al grupo 1, se le complementó la dieta con el producto evaluado (dos dosis de $60 \mathrm{~g}$ por semana por niño) por un periodo de seis meses. El grupo 2, siguió una alimentación normal. El alimento suministrado y el alimento ingerido diariamente cumplían con los requerimientos energéticoproteicos necesarios de un desayuno para niños en edad preescolar. Durante el tiempo que se desarrolló el estudio se observó la alimentación de los dos grupos, y además se impartieron pláticas sobre alimentación infantil a padres de familia (o tutores) y a los profesores de los jardines de niños; se desarrollaron talleres culinarios y sobre higiene alimentaria. A la mitad y al final del estudio, se realizó el segundo y tercer diagnóstico nutricional, respectivamente. Los tres diagnósticos nutricionales se compararon para determinar el efecto de la complementación alimentaria sobre el estado nutricional de los infantes, tal y como se describe en la sección de análisis estadísticos de datos. 
Análisis estadístico

Los resultados de la evaluación sensorial se analizaron mediante ji-cuadrado para determinar el nivel de agrado del producto; la comparación de los diagnósticos nutricionales se realizó mediante la prueba de Tuckey $(p<0.05)$, ambas pruebas utilizando el software Minitab® versión 16,0 para windows.

\section{RESULTADOS}

Evaluación sensorial

En la tabla 1, se muestran los resultados de la evaluación sensorial realizada con los niños de las tres comunidades. El análisis de ji-cuadrado de estos resultados muestran el alto nivel de agrado de los niños sobre el alimento elaborado a base de atún y soya $(p<0.01)$, se puede observar también que en las tres comunidades se presenta un alto nivel de agrado o preferencia de los niños sobre el producto, lo que indica su alto nivel de aceptabilidad, y bajo nivel de rechazo.

\section{Evaluación nutricional Indicador $\mathrm{P} / \mathrm{T}$}

En la figura 1, se presentan los resultados de los tres diagnósticos nutricionales efectuados, previo (1er Dx), a la mitad (2do Dx) y al final (3er Dx) del periodo de alimentación. En la figura se puede observar que previo a la complementación alimentaria y de acuerdo al indicador peso para la talla (peso/ talla), 51\% de los niños presentaban un estado nutricional normal, 35\% desnutrición leve (DL), 3\% desnutrición moderada (DM), 2\% desnutrición severa (DS), y $9 \%$ sobrepeso (SP) (de acuerdo a las tablas del Centro Nacional de Estadísticas de Salud (NCHS) de Estados Unidos de Norte América); lo que indica deficiencia de nutrientes en la alimentación de los

\section{TABLA 1}

Evaluación sensorial del alimento formulado a base de atún, soya y enriquecido con vitaminas y minerales.

Nivel de agrado

Me gusta

Ni megusta ni me disgusta

Me disgusta

Total

\section{J. Grajales}

$32 \mathrm{a}$

$3 b$

$1 \mathrm{~b}$

36
Jardín de niños Michoacán

$20 \mathrm{a}$

$1 \mathrm{~b}$

Ob

21
Tierra y Libertad

$55 a$

$6 \mathrm{~b}$

$3 c$

64

*Letras diferentes en la misma columna indican diferencias estadísticas significativas (ji-cuadrado, $\mathrm{p}<0.01$ ).

\section{FIGURA 1}

Resultados de los diagnósticos nutricionales de acuerdo al indicador $\mathrm{P} / \mathrm{T}$.

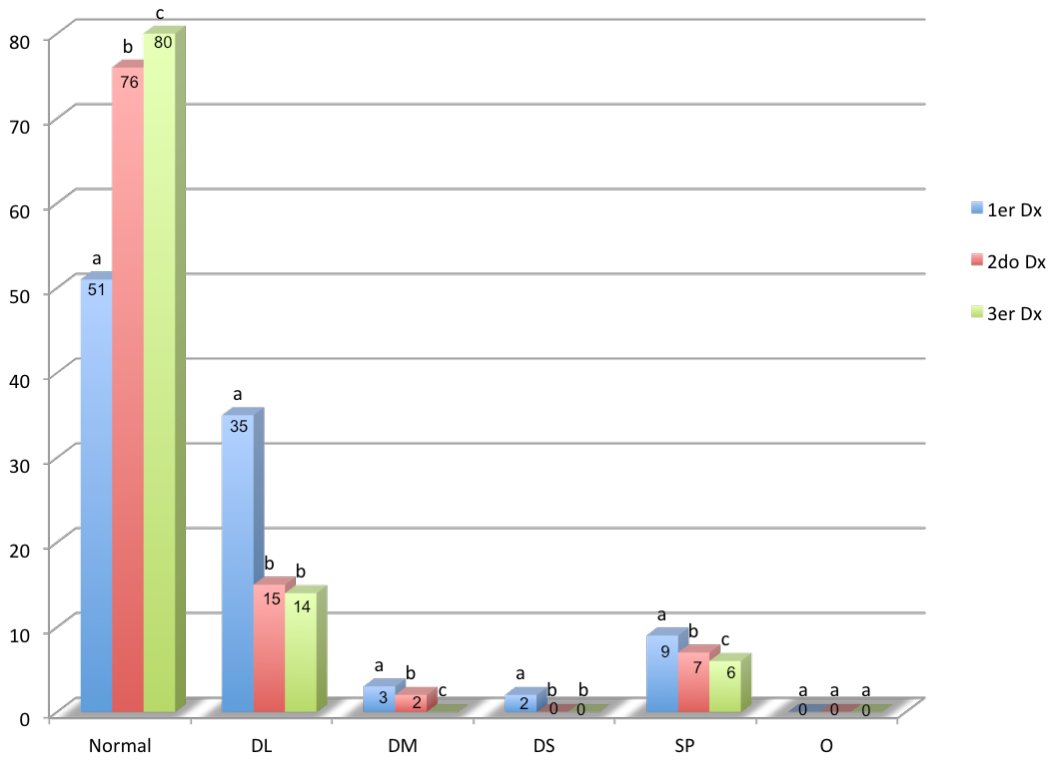

Letras diferentes por estado nutricional muestran diferencias estadísticas significativas (Tuckey, $\mathrm{p}<0.05$ ). 
preescolares, consecuentemente bajo peso y talla.

Al comparar la evaluación nutricional inicial, intermedia y final, se observó una diferencia estadística significativa (Tuckey, $\mathrm{p}<0,05)$, lográndose un aumento del $29 \%$ de los niños con estado de nutricional normal; los niños con DL, DM y DS, disminuyeron en un 21,3 y $2 \%$, respectivamente, resultados estadísticamente significativos (Tuckey, $\mathrm{p}<0,05$ ). Estos resultados evidencian un mejoramiento progresivo en la recuperación del estado nutricional de los preescolares que se les otorgó el alimento por un período de seis meses. La recuperación del estado nutricional de los niños se debe a que incrementaron su crecimiento, efecto que se puede atribuir a la ingesta de proteínas y vitaminas provenientes principalmente del atún y soya, componentes principales del alimento utilizado en la complementación alimentaria.

En la figura 2, de acuerdo al indicador peso para la edad (P/E) se puede observar que previo a la complementación alimentaria $43 \%$ de los niños presentaban un estado de nutrición normal, 39\% desnutrición leve (DL), y 18\% desnutrición moderada (DM); lo que indica deficiencia de nutrientes en los preescolares, consecuentemente bajo peso de acuerdo a la edad. Al comparar la evaluación nutricional inicial y final, se observó un aumento del $28 \%$ de los niños con estado nutricional normal; los niños que presentaban desnutrición leve disminuyeron en $12 \%$, y una disminución del $16 \%$ en niños con DM.

En la figura 3, de acuerdo al indicador talla para la edad (T/E) se puede observar que previo a la complementación alimentaria el $61 \%$ de los niños presentaban un estado nutricional normal, 32\% desnutrición leve (DL), y 7\% desnutrición moderada (DM). Al comparar la evaluación nutricional inicial y final, se observó un aumento del 19\% de los niños con estado de nutrición normal; la desnutrición leve disminuyó en 12\%, y una disminución del $7 \%$ en niños con DM.

\section{DISCUSIÓN}

El objetivo del presente estudio, fue evaluar el impacto nutricional de la complementación alimentaria de niños en edad preescolar (de 3 a 5 años y 9 meses) de tres comunidades marginadas del estado de Chiapas en México. La emaciación es definida como el bajo peso para la talla en niños, y es una medida de la desnutrición actual o aguda (10). Resultados similares al índicador P/T (figura 1) fueron presentados por Vela et al. (11) y Vela-Gutiérrez et al. (12), al evaluar el impacto nutricional de una papilla elaborada a base de frutas tropicales, y una galleta de harina trigo y soya, ambos alimentos adicionados con suero de leche en un grupos de niños en edad preescolar en dos comunidades de alta marginación del estado de Chiapas, México; respectivamente. En un estudio de intervención de consumo de leche fortificada con niños se demostró la mejora en peso (diferencia de 0,21 Kg/año) y aumento en talla (diferencia de 0,51 cm/año) comparado con el grupo control $(6,13)$. Los problemas de desnutrición específicamente baja talla (desmedro) en menores de cinco años afecta alrededor de 200 millones de niños en el mundo; en México, el problema afecta alrededor de 1,5 millones de niños de la misma edad (7). La malnutrición por deficiencia de micronutrientes (proteico-energética) continúa siendo la mayor carga de salud en países en desarrollo; globalmente es el único factor de riesgo para la enfermedad y muerte, con cientos de millones de mujeres embarazadas y niños jóvenes particularmente afectados. En los niños de países en desa-

\section{FIGURA 2}

Diagnósticos nutricionales de acuerdo al indicador P/E.

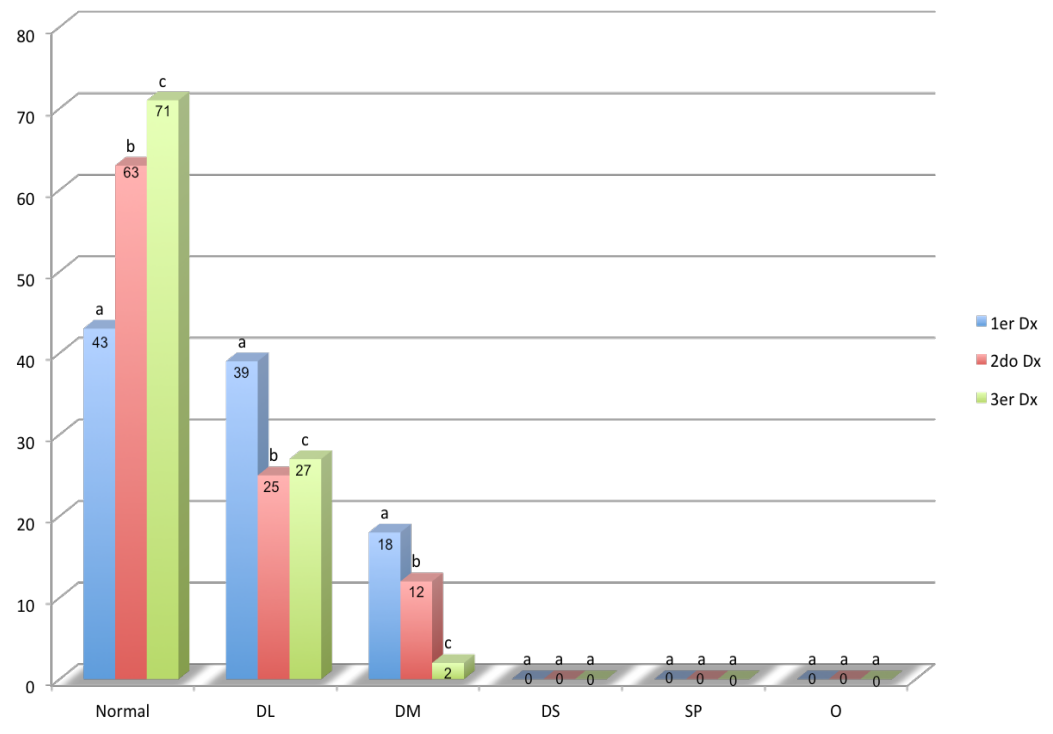

Letras diferentes por estado nutricional muestran diferencias estadísticas significativas (Tuckey, $\mathrm{p}<0.05$ ). 
rrollo, la desnutrición es considerada un problema de salud con prevalencias entre 4 a $46 \%$ con 1 a $10 \%$ de desnutrición sevéra (5). De acuerdo, a un estudio desarrollado por Josteen et al. (14) en los países bajos la prevalencia de niños con problemas de desnutrición fue 16.0\%; la desnutrición aguda fue $3.0 \%$, y la prevalencia de desnutrición crónica fue 13.8\%; y alrededor del $1.0 \%$ de los niños presentaron ambos problemas (desnutrición aguda y crónica). No se encontraron diferencias en desnutrición entre niños y niñas. Cuando existe déficit en el acceso y disponibilidad de alimentos, los niños son los primeros afectados, debido a la susceptibilidad a los problemas de salud y nutrición, más aún, los problemas de desnutrición son más frecuentes en zonas marginadas, tal es el caso de las comunidades Michoacán, Julián Grajales y Tierra y Libertad del municipio de Jiquipilas Chiapas, México. El estado nutricional de las personas en gran medida depende de su alimentación, condicionada por el acceso y la disponibilidad de los alimentos, y ligado a la baja o nula ingesta de alimentos de alta calidad proteica y calórica.

Un niño desnutrido tiene un escaso desarrollo muscular y posee mayor riesgo de presentar enfermedades crónicas en la edad adulta. Los efectos de una nutrición insuficiente no siempre son reversibles, puede recuperarse el peso y talla de los niños, pero la maduración del sistema nervioso no es recuperable. Un estudio desarrollado en Etiopía con 100 niños en edad escolar, se encontró una prevalencia de bajo peso del $21 \%$, más bajo al reportado previamente en el noreste de ese país, y al encontrado en el presente estudio (40\%). Otro estudio desarrollado en Mubai (India), reportó una prevalencia de desnutrición alta específicamente por bajo peso, los autores atribuyeron el problema a prácticas de complementación alimentaria deficiente, falta de conocimiento de las madres/cuidadores, malas prácticas higiénicas y bajo nivel socio-económico (3). Incluso con un óptimo desayuno, los niños podrían limitarse a no recibir una adecuada cantidad y calidad de alimentos antes de los seis meses de edad. Prácticas de lactancia apropiada y de alimentación complementaria son fundamentales para la supervivenvia, crecimiento y desarrollo de los niños. La lactancia frecuente también protege la salud del niño y el bienestar al reducir el riego de morbilidad y mortalidad en el niño en poblaciones afectadas (15). La baja talla es el reflejo de los efectos negativos acumulados a lo largo del tiempo. Este retardo en el crecimiento lineal ha tenido una franca disminución en la población preescolar en México, al pasar de $26.9 \%$ en 1998 a $13.6 \%$ en 2012 , es decir, una disminuación absoluta de 13.3 puntos porcentuales ( $p p)$ y una dismimución relativa de casi la mitad (49.4\%) (8).

En el presente estudio

\section{CONCLUSIONES}

- Se logró reducir el porcentaje de desnutrición leve y moderada de los niños a los que se les proveyó del alimento a base de atún y soya de las tres comunidades (Tierra y Libertad, Julian Grajales y Michoacán) de Chiapas en México durante seis meses, esto se debe a que la ingesta diaria de proteínas de alto valor biológico aumentó.

- El alimento mostró estadísticamente un alto nivel de agrado y aceptabilidad en los niños de las tres comunidades evaluadas.

\section{FIGURA 3}

Diagnósticos nutricionales de acuerdo al indicador T/E.

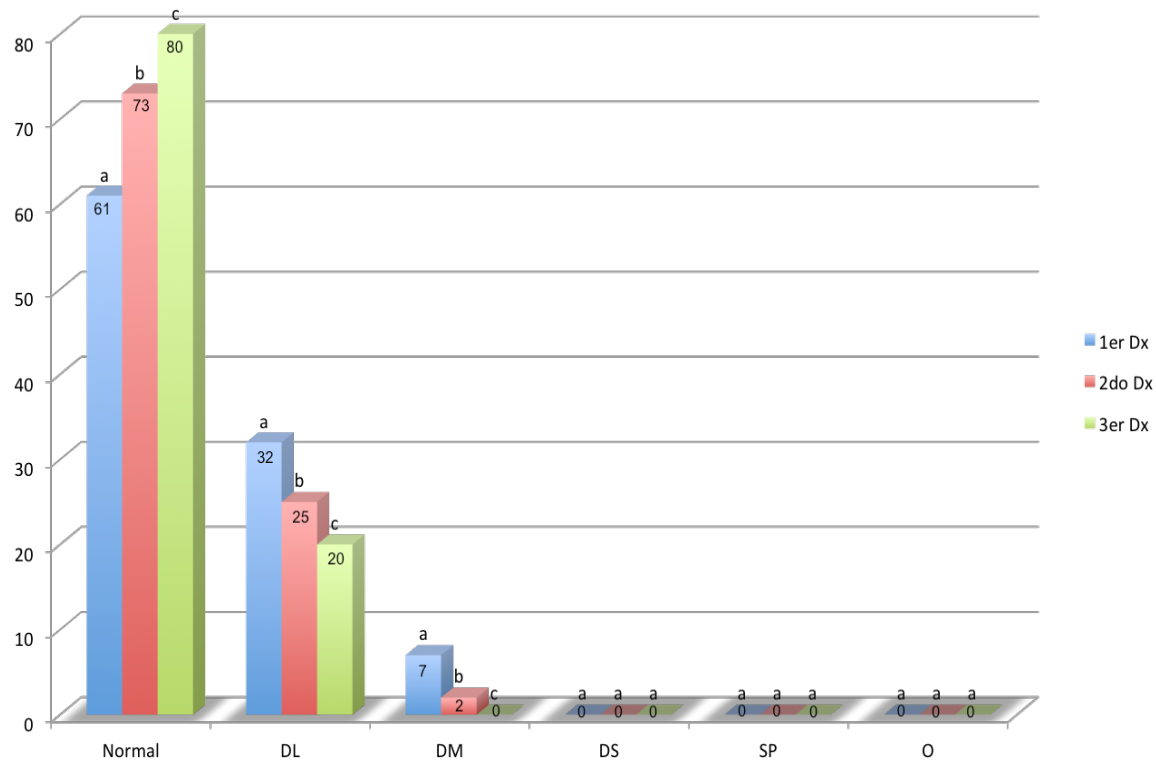

Letras diferentes por estado nutricional muestran diferencias estadísticas significativas (Tuckey, $p<0.05$ ). 


\section{RESUMEN}

El objetivo de este estudio fue determinar el impacto nutricional y sensorial de un alimento a base de atún y soya enriquecido con vitaminas y minerales en preescolares de tres comunidades de Chiapas, México. Estudio de intervención nutricional con una cohorte de seis meses. Se determinó el estado nutricional de los niños previo a la alimentación, a la mitad y al final de ésta; la muestra se conformó con los niños que presentaron problemas de desnutrición inicialmente; a los que se les complementó la alimentación durante seis meses. Se determinó el nivel de agrado del producto, con niños de las tres comunidades. Se encontraron evidencias significativas $(p<0.05)$ de un alto nivel de agrado del alimento, al comparar el estado nutricional obtenido al inicio, a la mitad y al final del estudio, se observó mejoras en el estado salud de los niños, al pasar de desnutrición severa o grave a normal.

Palabras clave: desnutrición; intervención alimentaria; niños preescolares; micronutrientes; alimento fortificado.

Agradecimientos: Los autores agradecen al Consejo $\mathrm{Na}-$ cional de Ciencia y Tecnología (CONACyT), a la Universidad de Ciencias y Artes de Chiapas, y a la Empresa Procesamiento Especializado de Alimentos de Chiapas, S.A. por el financiamiento otorgado para la realización del proyecto.

\section{BIBLIOGRAFÍA}

1. Mönckeberg B.F. Infant undernutrition: damage to the human capital, Rev Chil Nutr. 2014; 41(2):173-80.

2. Varea A. Malpeli A. Etchegoyen G. Vojkovic M. Disalvo L. Apesteguía M. Pereyras S. Pattín J. Ortale S. Carmuega E. González H.F. Short-Term Evaluation of the impact of a Food Program on the Micronutrient Nutritional Status of Argentinean Children under the Age of Six. Biol Trace Elem Res. 2011; 143:1337-48.

3. Lohia N. Udipi S.A. Infant and child feeding index reflects feeding practices, nutritional status of urban slum children, BMC Pediatrics 2014; 14:290-301.

4. Black A.P. Brinblecombe J. Eyles H. Morris P. Vally H. O'Dea K. Food subsidy programs and the health and nutritional status of disadvantaged families in high income countries: a systematic review, BMC Public Health 2012; 12: 1099-123.

5. Amare B. Moges B. Fantahun B. Tafess K. Woldeyohannes
D. Yismaw G. Ayane T. Yabutani T. Mulu A. Ota F. Kassu A. Micronutrient levels and nutritional status of school children living in northwest Ehiopia, Nutrition J. 2012; 11:108-16.

6. Eichler K. Wieser S. Rüthemann I. Brügger U. Effects of micronutrient fortified milk and cereal food for infants and children: a systematic review, BMC Public Health 2012; 12:506-19.

7. García-Parra E. Ochoa-Díaz-López H. García-Miranda R. Moreno-Altamirano L. Solís-Hernández R. Molina-Salazar $R$. Are there changes in the nutritional status of children of Oportunidades families in rural Chiapas, México? A cohort prospective study, J Health Population an Nutr. 2015; 35:1-9. DOI 10.1186/s41043-015-0038-5.

8. Gutiérrez J.P. Rivera-Dommarco J. Shamah-Levy T. Villalpando-Hernández S. Franco A. Cuevas-Nasu L. RomeroMartínez M. Hernádez-Ávila M. Encuesta Nacional de Salud y Nutrición (ENSANUT). Resultados Nacionales; Cuernavaca, México: Instituto Nacional de Slud Pública (MX), 2012.

9. Secretaría de Salud. NOM-031-SSA2, para la atención a la salud del niño, 1999.

10. Srivastava A. Mahmood S.E. Sristava P.M. Shrotriya V.P. Kumar B. Nutritional status of school-age children $-A$ scenario of urban slumns in India, Arch Public Health 2012; 70:8-16.

11. Vela G.G. Vargas G.F.M. Cortés P.E. López D.A., Flores G.L-E. López Z.E. E. Nutritional and sensorial impact of an infantile food (papilla) added with lactoserum. Rev Avances Seg Alim y Nutr. 2009; 1(1):31-6.

12. Vela-Gutiérrez G. Castro M.M. Caballero R.A. Ballinas D.J. Probiotic drink of lactoserum added with mangos pulp and almonds sensorially acceptable by adults majors, Rev. ReCiTelA 2012; 11(2): 8-20.

13. Shama-Levy T. Morales R.C. Amaya, C.C. Salazar C.A. Jiménez A.A. Méndez G.H.I. Effectiveness of a diet and physical activity promotion strategy on the prevention of obesity in Mexican school children, BMC Public Health 2012; 12:152-65.

14. Joosten K. Van der Velde K. Joosten P. Rutten H. Hulst J. Dulfer K. Association betwen nutritional status and subjective health status in chronically ill children attending special schools, Qual Life Res 2015; DOI 1.1007/s11136015-1130-4. 